\title{
Fractionation of homologous CD6 to CD60 cyclodextrin mixture by ultrafiltration and nanofiltration
}

\begin{abstract}
This paper investigates the membrane purification and fractionation of a mixture containing the homologous series of cyclodextrins CD6 to CD60 obtained by enzymatic conversion of starch. Three commercial polyamide thin film composite NF and UF membranes (HL, GH and GK from GE-Osmonics) were used for this purpose. In a first step, a binary mixture composed of glucose and heptacyclomaltose (b-cyclodextrin, CD7) was filtered to examine the separation performance of the studied membranes. A mathematical model based on mass balance was proposed for the simulation of the discontinuous diafiltration process assuming that the membrane separation performance is based on a sieving mechanism. A three stage diafiltration cascade (in retentate configuration) was then selected to fractionate the CD6i CD60 crude mixture. The experimental composition of the obtained permeate and retentate solutions in the targeted fractions (glucose, CD6ï CD8, CD9ï CD21, CD22ï CD60) fit well with the predicted data indicating that membrane process enables purification and fractionation of the homologous series of large ring CDs. Some discrepancies were however observed implying that other mechanisms such as coupled transport also took place. The most striking effect was the presence of glucose in the GK retentate possibly as a result of the formation of inclusion complexes with the large ring CDs.
\end{abstract}

Keyword: Large ring cyclodextrins; Fractionation; Ultrafiltration; Nanofiltration; Diafiltration 